\title{
Forgetting the fear
}

DOI:

10.1038/nrn2223

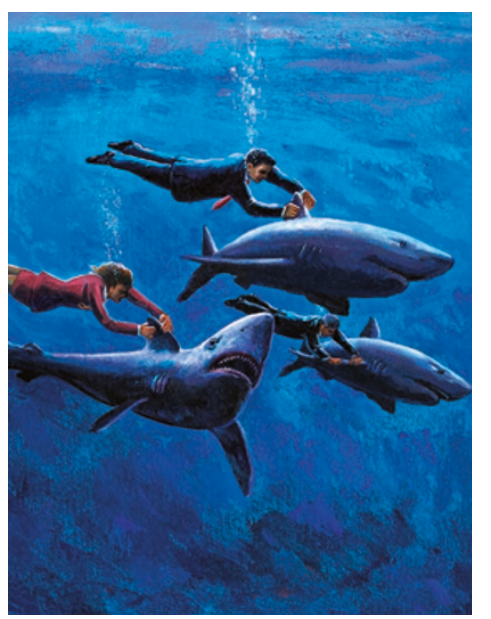

There are some memories one would rather forget. This is especially true for people who suffer from phobias or from post-traumatic stress disorder. Some memories can decrease and even disappear through a process called extinction, but the mechanisms that are involved are not known. Tsai and colleagues now show that a molecular pathway in the hippocampus that involves cyclindependent kinase $5(\underline{\mathrm{Cdk}} 5)$ regulates the extinction of contextual fear in mice.

When mice are exposed to an aversive stimulus in a neutral context, they develop fear for that context - this is called conditioned fear. In a subsequent 'extinction procedure' that consists of daily 3-minute long re-exposures to the context alone, the animals gradually become less afraid of the environment, as evidenced by reduced freezing.
The authors found that infusing a Cdk5 inhibitor into the hippocampus facilitated extinction if it was given up to 1 hour after the trials (giving it before had no effect), indicating that Cdk5 is involved in the consolidation of the new experience to long-term memory. Next, the authors increased Cdk5 activity by using transgenic mice in which forebrain expression of the Cdk5 activator p25 was switched on after fear conditioning. They found that increasing Cdk5 activity abolished extinction, whereas switching p25 off returned Cdk5 activity to baseline and re-instated extinction.

What happens to $\mathrm{Cdk} 5$ when extinction takes place? The authors measured the levels of Ckd5, and of its activator p35, and found that extinction was associated with a redistribution of $\mathrm{p} 35$ from the membrane to the cytosol of hippocampal lysates.

The location of Cdk5 and p35 is regulated by the GTPase Rac-1, and the authors found that infusion of a Rac-1 inhibitor into the lateral ventricles re-distributed hippocampal p35 and Cdk5 from the membrane to the cytosol, facilitating extinction. The extinction itself caused a reduction of membrane-associated Rac-1 activity in the hippocampus, indicating that extinction downregulates the Rac-1induced inhibition of Cdk5 and p35 redistribution in hippocampal cells.

Cdk5 and p35 can inactivate the kinase PAK-1 (which has been associated with actin re-arrangement, learning and synaptic remodelling) by phosphorylating it. The authors showed that extinction reduced this phosphorylation and thus upregulated PAK-1 activity. They also expressed dominant-negative PAK-1 in the hippocampus and showed that extinction was impaired, indicating a role for PAK-1 in this process. Next, the authors injected a Rac-1 inhibitor, which caused reduced binding of p35 to PAK-1 and increased PAK-1 activity in the cytosolic fraction of hippocampal lysates. Moreover, injecting a Cdk5 inhibitor reduced PAK-1 phosphorylation, increased its activity and caused its redistribution to the cytosol.

Together these findings suggest that, during extinction, decreased Rac- 1 activity in hippocampal cells causes a redistribution of Cdk5 and p35 to the cytosol, which then results in sequestering of $\mathrm{p} 35$ from PAK-1. This activates PAK-1 which then, through its involvement in the remodelling of synaptic circuits, facilitates extinction.

This study provides a molecular mechanism for the regulation of fear extinction. It opens up new avenues for the development of drugs that will target this pathway and which may, eventually, be used to extinguish specific memories.

Leonie Welberg

\section{ORIGINAL RESEARCH PAPER}

Sananbenesi, F. et al. A hippocampal Cdk5

pathway regulates extinction of contextual fear. Nature Neurosci. 10, 1012-1019 (2007) 